Editorial

\title{
Acknowledgment to Reviewers of Fluids in 2021
}

\section{Fluids Editorial Office}

Citation: Fluids Editorial Office. Acknowledgment to Reviewers of Fluids in 2021. Fluids 2022, 7, 57. https://doi.org/10.3390/fluids7020057

Published: 26 January 2022

Publisher's Note: MDPI stays neutral with regard to jurisdictional claims in published maps and institutional affiliations.

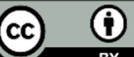

Copyright: $\left({ }^{\circ} 2022\right.$ by the authors. Licensee MDPI, Basel, Switzerland.

This article is an open access article distributed under the terms and conditions of the Creative Commons Attribution (CC BY) license (https://creativecommons.org/licenses/by/4.0/).

MDPI AG, St. Alban-Anlage 66, 4052 Basel, Switzerland

Rigorous peer-reviews are the basis of high-quality academic publishing. Thanks to the great efforts of our reviewers, Fluids was able to maintain its standards for the high quality of its published papers. Thanks to the contribution of our reviewers, in 2021, the median time to first decision was 17 days and the median time to publication was 43 days. The editors would like to extend their gratitude and recognition to the following reviewers for their precious time and dedication, regardless of whether the papers they reviewed were finally published:

Abbasnia, Arash

Abdelkarim, Aydi

Abdelsalam, Sara I.

Abdullah, Nur Azam

Abiev, Rufat

Agullo, Olivier

Ahmed, Anwar

Aijaz, Saima

Airiau, Christophe

Akbar, Muhammad K.

Alam, Jahrul

Alam, Miah Md Ashraful

Alcántara-Ávila, Jesús Rafael

Alexandrakis, George

Alexey, Kiverin

Ali, Akram

Ali, Naseem

Alihussein, Hussein

Aliyu, Aliyu

Al-Mdallal, Qasem M.

Amadori, Marina

Amaechi, Chiemela Victor

Amani, Ahmad

Aminoroayaie Yamini, Omid

Anand, Nadish

Angiulli, Giovanni

Anjos, Gustavo R.

Annenkov, Sergei

Aouane, Othmane

Aricò, Costanza

Arjunan, Arun

Artemov, Mikhail A.

Ashrafi, Nariman

Attal, Nitesh Omprakash
Azamathulla, Hazi M.

Azarova, Olga A.

Azhikodan, Gubash

Bade, Mehar

Balachandran, Balakumar

Baliti, Jamal

Baltazar, Luís Gonçalo Correia

Ban, Takahiko

Barata, Jorge Manuel Martins

Barati, Reza

Barbaro, Giuseppe

Barsi, Dario

Bartosik, Artur

Basan, Elena

Basuray, Sagnik

Batikh, Ahmad

Battista, Nicholas

Bautista, Oscar

Behzadinasab, Masoud

Beji, Serdar

Bekkioui, Naoual

Bellur, Kishan

Benetazzo, Alvise

Benim, Ali Cemal

Benkhaldoun, Fayssal

Bergmann, Michel

Bhowmik, Palash

Bianchini, Roberta

Bieniek, Andrzej

Bikić, Siniša

Bilican, Doga

Bilotta, Giuseppe

Bitner-Gregersen, Elzbieta M.

Bobusch, Bernhard C. 
Bois, Guillaume

Borojeni, Azadeh A.T.

Breitsamter, Christian

Brufau, Pilar

Bruschewski, Martin

Bryś, Andrzej

Budwig, Ralph

Buick, James

Burmasheva, Natalya

Bustamante, Eliseo

Buttà, Paolo

Calil, Paulo Rezende

Cambon, Claude

Cannata, Giovanni

Cantrak, Djordje

Capone, Alessandro

Capuano, Francesco

Caridade, Pedro Jorge Dos Santos Branco

Caulfield, Colm

Cavaco Mendes, Mário José Gonçalves

Chakrabarti, Brato

Chalecki, Marek

Chan, Cho Lik

Chan, Edward

Chang, Chih-Wen

Chávez-Modena, Miguel

Chen, Bang-fuh

Chen, Chengpeng

Chen, Dyi-Cheng

Chen, Jui-Sheng

Chen, $\mathrm{Ru}$

Chen, Yang

Chernov, Ilya A.

Chernyshov, Mikhail

Chertovskikh, Roman

Chesneau, Christophe

Cintolesi, Carlo

Cioncolini, Andrea

Cisterna, Pedro

Coelho, Rodrigo C. V.

Cornejo, Ivan

Cornejo, Pablo

Coronado-Hernández, Oscar E.

Corvaro, Sara

Cosoiu, Costin Ioan

Costa, Pedro Simões

Croce, Giulio

Cruzeiro, Ana Bela

Cuenot, Bénédicte

Czarnota, Robert

D'angola, Antonio
Da Cunha Lima, André T.

Dahl, Jason

Davidy, Alon

De Leo, Francesco

De Oliveira, Pedro Magalhães

De Vanna, Francesco

Deepak, Doreswamy

Del Giudice, Francesco

Del Rio Oliveira, Santiago

Deng, Xi

Deshpande, Rahul

Dessi, Claudia

Dhariwal, Rohit

Dharmarathne, Suranga

Di Bernardino, Annalisa

Di G. Sigalotti, Leonardo

Díaz-Hernandez, Gabriel

Dimitrijević, Milan S.

Dong, Kejun

Dongare, Pratiksha

Dreglea, Aliona Dreglea

Drimer, Nitai

Drosatos, Panagiotis

Duda, Daniel

Đurin, Bojan

Edoh, Ayaboe

Egolf, Peter W.

Eid, Mohamed Rabea

El Baroudi, Adil

Elhaj, Murtada A.

Elías-Zúñiga, Alex

Emami, Noushin

Ene-Iordache, Bogdan

Engwirda, Darren

Ershkov, Sergey

Evans, Jonathan

Fabrizio, Pistani

Falkowska, Ewa

Fan, Dixia

Fang, Ruixian

Favretti, Marco

Fawwaz Alrebei, Odi

Febres, Gerardo

Fedorov, Ruslan V.

Fedorov, Vladimir E.

Feng, Haidong

Fernandez Oro, Jesus Manuel

Fernández, Carlos Vilas

Ferrás, Luís L.

Feuchter, Claus

Fezi, Kyle 
Figwer, Jarosław

Fisher, Alexander W.

Flack, Karen A.

Fossati, Marco

Fraga, Bruño

Fragos, Vassilios

Franci, Alessandro

Freund, Andreas

$\mathrm{Fu}$, Dong

Fuentes-Pérez, Juan Francisco

Gajjar, Jitesh

Gallagher, Meurig T.

Galvin, Kevin

Gao, Linyue

García García, F. Javier

Garduño, Isaías E.

Gąsiorowski, Dariusz

Gavaises, Manolis

Ge, Wenjun

Gerontidou, Maria

Gevari, Moein Talebian

Gharali, Kobra

Ghorbani, Morteza

Gilja, Gordon

Ginzburg, Irina

Girfoglio, Michele

Gissen, Abraham

Glasser, Leslie

Gomez, Ricardo S.

Gonçalves, Helena M. R.

Goring, Derek

Goswami, Shubhodeep

Goubergrits, Leonid

Gratien, Jean-Marc

Griffin, James

Grucelski, Arkadiusz

$\mathrm{Gu}$, Yingsong

Gué, Emilie

Guo, Hanliang

Guo, Weijin

Guzmán-Lastra, Francisca

Halila, Gustavo Luiz Olichevis

Halse, Karl Henning

Han, Lianfu

Hao, Nanjing

Hayashi, Kosuke

Hegab, Hussien

Heiden, Bernhard

Heisel, Michael

Helgadóttir, Ásdís

Henderson, Diane
Hernández, Rodrigo H.

Hernández-Fontes, Jassiel Vladimir

Hirata, Silvia

Hitimana, Emmanuel

Hlaváčová, Irena M.

Hoenders, Bernhard J.

Horwitz, Jeremy

Hossain, Akter

Houari, Ameur

Hssikou, Mohamed

$\mathrm{Hu}$, Guang

Huete, César

Hussanan, Abid

Hussein, Ahmed Kadhim

Hynčík, Luděk

Ilieva, Galina

Inagaki, Atsushi

Ioannis, Sarris

Ionete, Eusebiu Ilarian

Isoz, Martin

Issler, Dieter

Isvoranu, Dragos

Iuppa, Claudio

Ivers, David

Iwaniszyn, Marzena

Iyer, Prahladh S.

Izonin, Ivan

Jain, Abhishek

Jain, Kartik

Jakšić, Olga

Jault, Dominique

Jayathilake, Pahala Gedara

Jiang, Nan

Juez, Carmelo

Kagemoto, Hiroshi

Kahnamouei, Jalal Taheri

Kala, Petr

Kanaris, Athanasios G.

Kanbur, Barış Burak

Kang, Dong Gu

Kapyrin, Ivan V.

Kara, Kursat

Karbaschian, Ali

Kari, Leif

Karim, Md. Rezwanul

Kartushinsky, Aleksander

Kasper, Robert

Katsaounis, Georgios M.

Kauffman, Justin

Kawamoto, Shoichi

Kefayati, Gholamreza 
Keramaris, Evangelos

Khalique, Chaudry

Khan, Abdul Samad

Khan, Md. Shakhaoath

Kiani, Keivan

Kianvashrad, Nadia

Kieu, Chanh

Kiliçman, Adem

KIM, Jeong Soo

Kiyama, Akihito

Kobus, Artur

Kochurin, Evgeny A.

Koklu, Mehti

Kolimas, Łukasz

Kolotova, Daria S.

Kong, Ran

Konovalov, Sergey

Kontakiotis, George

Kõrgesaar, Mihkel

Koscak, Peter

Kotov, Mikhail

Kovács, Róbert

Kraposhin, Matvey

Kravtsov, Sergey

Krieger, Michael

Krishan, Gopal

Krivovichev, Gerasim Vladimirovich

Krometis, Justin

Kruzel, Marcin

Kuchumov, Alex

Kuehl, Joseph

Kulawik, Adam

Kumamaru, Hiroshige

Kumar, Krishna

Kumar, Praveen

Kurashina, Ryosuke

Kurkina, Oxana E.

Kurtulus, Dilek Funda

Kuznetsov, Nikita M.

Kwiatkowski, Mirosław

Kwon, Beomjin

Koutandos, Evangelos V.

Laca Pérez, Amanda

Lai, Huilin

Laity, Peter R.

Lampropoulou, Paraskevi

Lavoie, Pierre

Lee, Chulmin

Lee, Hee Joon

Lee, Myoungkyu

Leftwich, Megan
Lemoine, Jean Philippe

León, Alejandro

Lerch, Andre

Lewandowski, Michal T.

Li, Deyou

Li, Jian

Li, Wanai

Li, Xiaocan

Li, Zhi

Liakos, Anastasios

Liberge, Erwan

Ligęza, Paweł

Ling, Bowen

Lisicki, Maciej

Liu, Jia

Liu, Jih-Shun

Liu, Shuhong

Ljubicic, Robert

Longatte-Lacazedieu, Elisabeth

Loureiro, Felipe Dos Santos

Lozano, Antonio

Lyubimova, Tatyana

Lyubimova, Tatyana P.

Ma, Shenglin

MacTaggart, David

Madhu, Kalyanasundaram

Madjarević, Damir

Maldonado, José Miguel

Malikan, Mohammad

Malvè, Mauro

Mancini, Marco

Mancini, Simone

Manikantan, Harishankar

Manservisi, Sandro

Maqueda, Gregorio

Marcos, Marcos

Maria Giuseppina Chiara, Nestola

Maric, Tomislav

Mariotti, Alessandro

Marquet, Olivier

Marsano, Davide

Marseglia, Guido

Martynenko, Sergey I.

Maruthamuthu, Murali

Maryshev, Boris

Marzouk, Osama

Masoud, Hassan

Masuda, Hayato

Mathew, Bobby

Mathioulakis, Demetri

Matsui, Hiroaki 
Mauro, Francesco

Mayeli, Peyman

Mazzanti, Valentina

McClure, Dale

McNamee, Antony P.

Meekins, Dave

Mehrizi, Abbasali Abouei

Mei, Lanju

Melton, Latunia P.

Michael, Berhanu

Michailides, Constantine

Michalski, Jacek

Mihai, Cruceru

Mikhailov, Evgeny

Mileva, Elena

Miller, Laura

Mizerski, Krzysztof Andrzej

Modigell, Michael

Mohammadian, Majid

Mongil-Manso, Jorge

Mori, Masaaki

Moriguchi, Shuji

Mosavati, Babak

Moussavi Tayyebi, Saeid

Muller, Milos

Muñoz, Daniel Horna

Murea, Cornel Marius

Mustafaoglu, Nur

Nabi, Saleh

Nachbin, Andre

Nag, Preetom

Narciso, Javier

Navas-Montilla, Adrian

Nazari, Behzad

Nedelcu, Dorian

Neunteufel, Michael

$\mathrm{Ng}$, Hoi Dick

Nguyen, Hoa

Nguyen, Huu Phu

$\mathrm{Ni}$, Qing

Nikhil, Nedumpallile Vasu

Nivedita, Nivedita

Nocivelli, Lorenzo

Noël, Romain

Nourian, Amir

Novoselov, Alex

Nowakowski, Bernard

Nowakowski, Jacek

Nunna, Bharath Babu

O'Rear, Edgar

Oettingen, Mikolaj
Ohm, Laurel

Oishi, Cássio

Okuducu, Mahmut Burak

Olcay, Ali Bahadır

Oliveira Panão, Miguel R.

Oliveira, Santiago Del Rio

Oppelstrup, Jesper

Orman, Lukasz

Ostanek, Jason

Ostermann, Florian

Owen, Benjamin

Owen, Herbert

Pakhomov, Maksim

Palade, Liviu Iulian

Panapakidis, Ioannis P.

Panas, Andrzej

Pant, Chandra Shekhar

Paprota, Maciej

Parard, Gaëlle

Parisse, Jean-Denis

Parsazadeh, Mohammad

Pasculli, Antonio

Pasha, Amjad Ali

Patel, Harshkumar

Patmonoaji, Anindityo

Patra, Bishnubrata

Paul, Akshoy Ranjan

Peer, Petra

Peixinho, Jorge

Peković, Ognjen M.

Pelinovsky, Efim

Pellegrini, Riccardo

Pendar, Mohammad-Reza

Pérez-García, José

Pergher, Sibele B. C.

Perov, Nikolai

Peruzzi, Cosimo

Petrik, Máté

Petrosyan, Arakel

Phu, Nguyen Minh

Piedra, Saul

Pigazzini, Riccardo

Pimpin, Alongkorn

Pitas, Charalampos N.

Pitt, Jonathan S.

Polivanov, Pavel A.

Pop, Nicolae

Possenti, Luca

Postnikov, Eugene

Pozorski, Jacek

Pralits, Jan Oscar 
Premkumar, M.

Premnath, Kannan N.

Prosviryakov, Evgenii

Pucciarelli, A.

Quaranta, Emanuele

Radhakrishnan, Kanmaniraja

Rädle, Matthias

Rahbari, Iman

Rahimzadeh, Amin

Rajaram, Dushhyanth

Rajski, Krzysztof

Rashad, Ahmed M.

Rashidi, Mohammad Mehdi

Ravipati, Srikanth

Rees, Andrew

Rees, D. Andrew S.

Reidenbach, Matthew

Reinaud, Jean

Reiss, Julius

Ren, Qinlong

Rezaian, Elnaz

Rhi, Seok-Ho

Riaz, Amir

Ricci, Alessio

Ricciardi, Guillaume

Rieck, Jan Klaus

Rodriguez, Ivette

Romano, Elio

Romanò, Francesco

Roşca, Alin V.

Rosic, Bojana

Rossi, Julio Daniel

Rosso, Fabio

Rubenstein, David A.

Rudko, Mykhailo

Ryota, Tsubaki

Ryzhov, Evgeny

Saavedra, Jorge H.

Saccomandi, Paola

Safaei, Mohammad Reza

Saghi, Hassan

Saha, Renata

Saha, Rupayan

Salahudeen, Mohamed

Salcedo-Castro, Julio

Samarzija, Ivana

Sarmadian, Alireza

Sarris, Ioannis

Sattin, Fabio

Sawicki, Jerzy

Scappaticci, Lorenzo
Scargiali, Francesca

Schiel, David

Schinas, Orestis

Scholle, Markus

Schweitzer, Marc Alexander

Sciacovelli, Luca

Semlitsch, Bernhard

Serra, Marco

Shafiq, Anum

Shahnam, Mehrdad

Shamim, Jubair A.

Shang, Delei

Shao, Songdong

Sharan, Nek

Sheremet, Mikhail

Shevchenko, Igor

Shevchuk, Igor V.

Shi, Aiming

Shirvani, Khosro A.

Shorstkii, Ivan

Shrivastava, Shamit

Shugan, Igor

Sidharth, G. S.

Sikora, Małgorzata

Silantyeva, Elena A.

Simate, Isaac N.

Simo Tagne, Merlin

Singh, Abhinendra

Sivasubramanian, Jayahar

Skripov, Pavel

Smoleń, Andrzej

Smyk, Emil

Sohn, Jung Woo

Sonnino, Giorgio

Specklin, Mathieu

Srivastava, Shubham

Stavroulakis, Georgios E.

Steijl, Rene

Stössel, Achim

Streckiene, Giedre

Strušnik, Dušan

Strzelewicz, Anna

Sutyrin, Georgi G.

Svorcan, Jelena

Szafran, Roman G.

Szasz, Robert-Zoltan

Szewczyk, Roman

Tabri, Kristjan

Tafarojnoruz, Ali

Takeishi, Naoki

Talebizadeh Sardari, Pouyan 
Tangermann, Eike

Tantos, Christos

Tanveer, Anum

Tarokh, Ali

Tassi, Emanuele

Tassone, Alessandro

Tavallaeinejad, Mohammad

Teimourian, Amir

Terashima, Hiroshi

Terziev, Momchil

Testoni, Raffaella

Thapa, Mishal

Theillard, Maxime

Thompson, Roney

Tian, Fang-Bao

Tiselj, Iztok

Torlo, Davide

Torres, Claudio E.

Triki, Ali

Trimulyono, Andi

Truchot, Benjamin

True, Aaron

Tucny, Jean-Michel

Ugarte, Orlando

Uscilowska, Anita

Ushida, Akiomi

Uzunoglu, Emre

Vachaparambil, Kurian

Valero, Mario M.

Vargas-Chable, Pedro

Vasquez, Paula

Veldman, Arthur E. P.

Venegas, Javier Freire

Venturini, Paolo

Viccione, Giacomo

Vieru, Dumitru

Villasmil, Larry A.

Viswanathan, Harish

Vita, Francesco De

Wacławczyk, Marta

Walker, Benjamin
Wang, Chuanjin

Wang, Guanyi

Wang, Jin

Wang, Jindong

Wang, Po-Ching

Wang, Shan

Wauters, Jolan

Weiss, Julien

Whalen, Edward A.

Wilczynski, Krzysztof

Wołosz, Krzysztof J.

Worwag, Malgorzata

$\mathrm{Xi}$, Huan

$\mathrm{Xu}, \mathrm{Ao}$

$\mathrm{Xu}$, Jinze

Yalcin, Huseyin Cagatay

Yan, Shiqiang

Yang, Chen

Yang, Dan

Yavuz, Mehmet

You, Donghyun

Yousefi, Kianoosh

Zafar, Azhar

Zahoor Raja, Muhammad Asif

Zaitsev, Andrey

Zang, Bin

Zangeneh, Rozie

Zarepoor, Masoud

Zawala, Jan

Zhan, Lu

Zhang, Feixia

Zhang, Kai

Zhang, Mingji

Zhao, Jianan

Zhou, Haichao

Zhu, Xiaowei

Zong, Haibo

Zou, Ling

Zoubeik, Mohamed

Zunino, Paolo

Zurauskiene, Nerija 